\title{
RECHERCHES SUR LA COMPOSITION DES PRINCIPALES ESPECES DE POISSONS LE PLUS LARGEMENT UTILISÉES DANS L'ALIMENTATION DES POPULATIONS OUEST-AFRICAINES; ÉTUDE SUR L'INFLUENCE DES TECHNIQUES TRADITIONNELLES DE CONSERVATION
}

H. DUPIN et A. WANE

O. R. A. N. A., Dakar.

En bien des régions du Sénégal, le poisson constitue l'apport le plus important en protéines animales. Le poisson est consommé tantôt frais, tantôt après transformation (plus de $20 \%$ de la production).

Notre étude a essentiellement pour but d'évaluer les principales modifications physicochimiques provoquées par les procédés traditionnels de traitement du poisson en vue de sa conservation. Les difficultés d'écoulement du poisson frais, abondant pendant le saison de pêche, a conduit en effet à des formes variées de conservation, largement utilisées, solidement implantées dans la pratique culinaire africaine, car adaptées au goût des populations et à leur pouvoir d'achat.

Nous avons étudié :

- Le fermenté séché (guedj).

- Le braisé fumé séché (kéthiakh).

- Le grillé, fumé (métora).

Il nous a semblé nécessaire d'effectuer des analyses sur les mêmes espèces avant et après transformation pour mieux dégager les éventuelles modifications. Nous nous sommes intéressés surtout aux aspects suivants :

- humidité;

- différentes formes azotées;

- lipides;

- vitamine $\mathrm{B} 1$ et $\mathrm{B} 2$;

- histamine.

RÉSULTATS E'T DISCUSSION

Humidité.

Le caractère artisanal, l'absence de législation et le rythme d'écoulement concourent pour aboutir à des taux d'humidité très variables après les opérations de transformation $(10$ à $40 \%)$. 
Ces taux, le plus souvent élevés, diminuent la durée de conservation en favorisant d'une part les processus bactériens de dégradation des protéines conduisant à accroître les formes d'N non assimilables (bases volatiles)et d'autre part à faciliter le parasitage par les dermestes (larves et adultes qui sont à l'origine de pertes pouvant atteindre $15 \%$ en une dizaine de jours). Dans le tableau 1 nous donnons les taux extrêmes d'humidité que nous avons observés.

\section{TABleat 1}

\begin{tabular}{|c|c|c|c|}
\hline ESPĖCES & ETAT & HUMIDITÉ & PHOTIDES \\
\hline \multirow[t]{3}{*}{ Congre } & frais & 78,3 & 19,5 \\
\hline & guedj & 32,0 & 63,6 \\
\hline & guedj bien séche & 13,9 & 77,3 \\
\hline \multirow[t]{3}{*}{ Dorade rose } & frais & 77,3 & 19,6 \\
\hline & guedj & 38,5 & 39,9 \\
\hline & guedj bien séché & 11,3 & 77,3 \\
\hline \multirow[t]{3}{*}{ Silure } & frais & 76,3 & 19,4 \\
\hline & métora & 34,2 & 56,3 \\
\hline & métora bien préparé & 17,0 & 78,1 \\
\hline \multirow[t]{3}{*}{ Fausse norue } & frais & 76,8 & 19,7 \\
\hline & guedj & 35,8 & 42,9 \\
\hline & guedj bien séché & 15,2 & 76,2 \\
\hline \multirow[t]{2}{*}{ Sardinelles } & frais & 76,0 & 19,4 \\
\hline & kéthiakh & 19,9 & 68,7 \\
\hline
\end{tabular}

Les formes azotées.

Nous n'avons pas cru devoir convertir l'N total des produits de transformation en protéines en raison d'une proportion importante d' $\mathrm{N}$ non protéique (base volatiles totales, $\mathrm{N}$ formol). Le facteur de conversion 6,25 encore valable pour le poisson frais, n'est plus applicable. Si pourtant nous l'employons, les produits bien séchés renfermeraient jusqu'à plus de $70 \%$ de protéines, c'est-à-dire autant que les farines de poisson qui posent tant de problèmes en alimentation humaine.

\section{Bases volatiles totales.}

Elles sont essentiellement constituées de NH3. Elles rendent compte du degré de fermentation en fin de préparation. Leur évaluation dans les salés séchés importés du Congo ex-belge a permis à ce pays d'établir une base de législation.

Nous avons constaté que leur taux diminue en fonction du temps de conservation pour les produits bien séchés (tableau 2). Ceci peut remettre en cause le critère de bonne préparation basé sur leur estimation.

\section{N Formol.}

Rend compte de la teneur en amino-acides libres. Son évaluation en fonction du temps de conservation ne nous a pas permis d'en dégager des conclusions. Notons seulement que ses proportions peuvent atteindre $8,5 \%$ de l' $N$ total (tableau 3 ). 
Tableau 2

Variations des bases volatiles totales en fonction du temps de conservation en $\mathrm{mg} \mathrm{p} .100 \mathrm{~g}$

\begin{tabular}{l|r|r|r}
\hline \multicolumn{1}{c|}{ ESPÈces } & $\begin{array}{c}\text { FIN DE } \\
\text { PRÉPARATION }\end{array}$ & $\begin{array}{c}\text { AU HOL'T } \\
\text { v'UN MOIS }\end{array}$ & $\begin{array}{c}\text { AU BOUT } \\
\text { DE DEUX MOIS }\end{array}$ \\
\hline Congre (Kéthiakh) & 353 & 210 & 144 \\
Dorade rose (Guedj) & 485 & 375 & 282 \\
Silure (Métora) & 491 & 143 & 142 \\
Fausse morue (Guedj) & 427 & 269 & 146 \\
Ethmalose (Kéthiakh) & 257 & 102 & 73 \\
\hline \hline
\end{tabular}

\section{Urée.}

Nous n'avons pas trouvé trace de cette substance malgré les fortes fermentations bactériennes. Du reste nos échantillons n'appartiennent pas aux espèces cartilagineuses.

\section{Lipides.}

Les poissons étudiés se sont révélés maigres dans l'ensemble, Cependant une classification précise nécessite une étude assez longue. Des baisses lipidiques se produisent au cours de la transformation. Ceci est plus marqué pour le métora de silure en raison du traitement thermique de durée plus ou moins longue. En voici les variations calculées par rapport à $100 \mathrm{~g}$ de matière sèche.

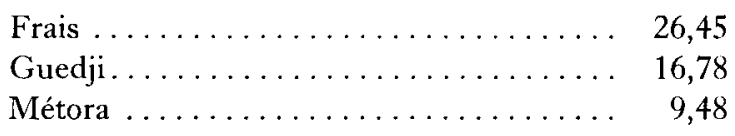

Le même calcul sur les fermentés séchés nous donne les valeurs suivantes :

$$
\text { Frais Fermenté séché }
$$

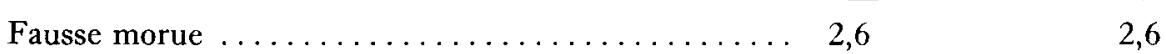

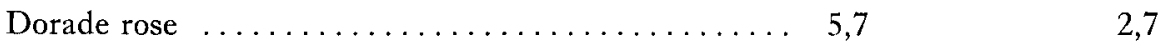

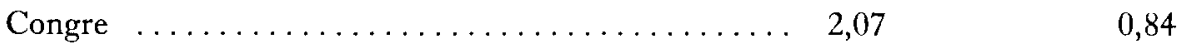

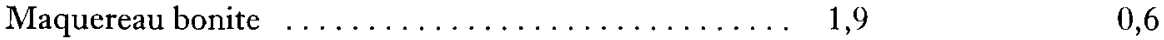

Pagre..................... $6,6 \ldots \ldots$ 4,7

Y aurait-il baisse par simple séchage au soleil ou modification chimique (oxydation) des lipides? Il ne nous est pas possible, pour le moment de répondre à cette question.

\section{Vitamines.}

Les vitamines $\mathrm{B} 1$ et $\mathrm{B} 2$ ont surtout retenu notre attention. Elles sont susceptibles en effet de diminuer au cours des opérations de transformation : séchage, traitement 
H. DUPIN, A. WANE

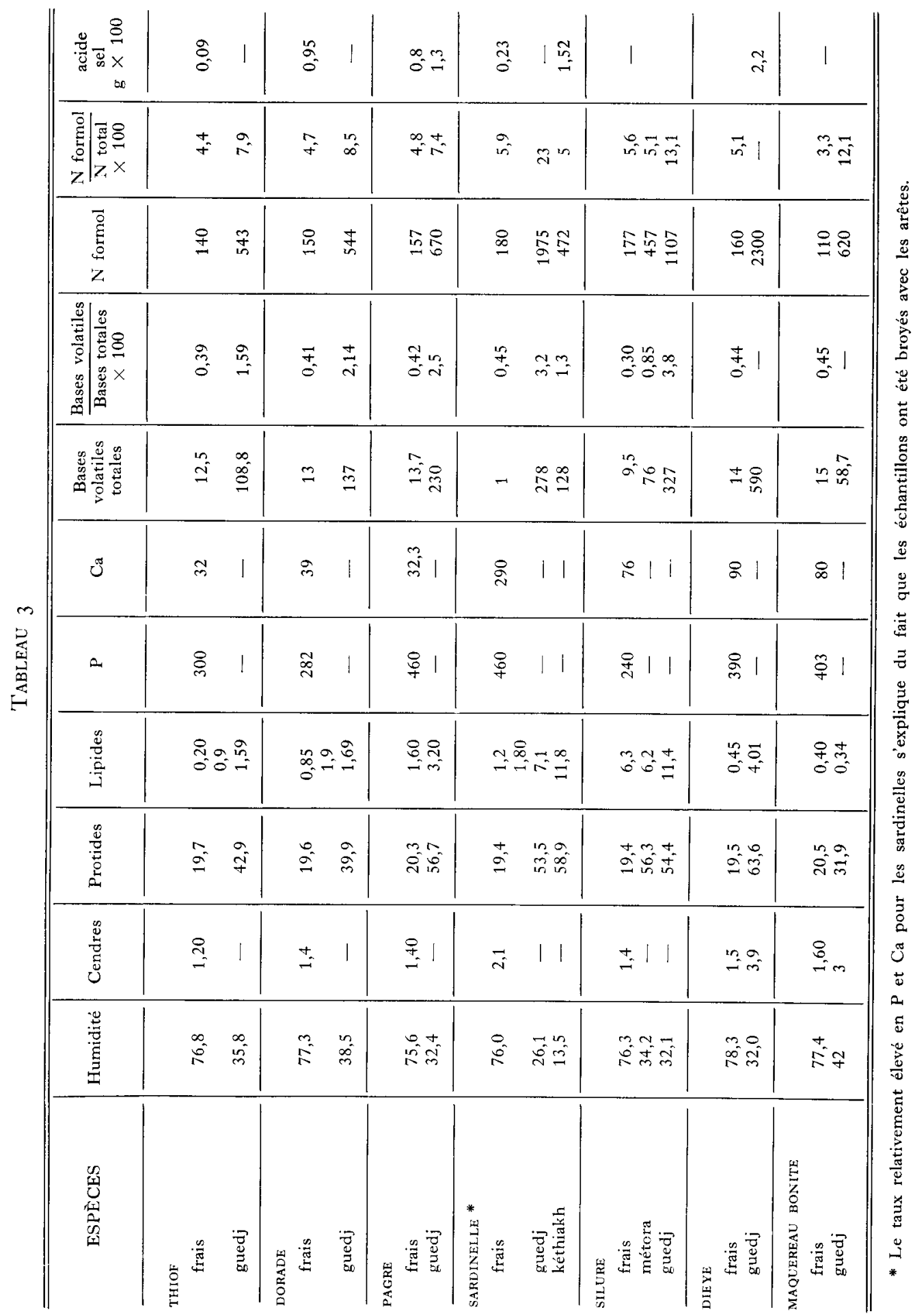


thermique. Nos analyses sont en cours. Nous donnons à titre d'exemple, deux résultats pour la vitamine B1.

\begin{tabular}{|c|c|c|c|}
\hline ESP ÈCE & ÉTAT & B1 en mcg p. $100 \mathrm{~g}$ & $\begin{array}{l}\text { B2 en mcg p. } 100 \mathrm{~g} \\
\text { de matière sèche }\end{array}$ \\
\hline $\begin{array}{l}\text { Fausse morue } \\
\text { Fausse morue } \\
\text { Silure } \\
\text { Silure }\end{array}$ & $\begin{array}{l}\text { frais } \\
\text { guedji } \\
\text { frais } \\
\text { métora }\end{array}$ & $\begin{array}{l}10 \\
30 \\
20 \\
58\end{array}$ & $\begin{array}{l}44 \\
34 \\
84 \\
64\end{array}$ \\
\hline
\end{tabular}

\section{Histamine.}

Les fermentations bactériennes et l'autolyse enzymatique mises en évidence par l'augmentation dans de fortes proportions des bases totales volatiles et de l'azote formol nous ont conduits à rechercher des amines toxiques susceptibles de se produire, singulièrement l'histamine. Il semble prouvé que cette amine provient de la décarboxylation de l'histidine par les bactéries contenant l'histidine-décarboxylase : B aminophilus, B coli, anéorobies (Van Veen et Latuasan). D'après ces auteurs, $20 \%$ de l'histidine peuvent être transformées en histamine au bout de 48 heures et à $30^{\circ} \mathrm{C}$.

Le dosage de l'histamine par voie chimique selon la méthode de Parkhurst A. Shore, A. Burkhalter et V. H. Cohn nous a fourni sur quelques échantillons les taux cidessous.

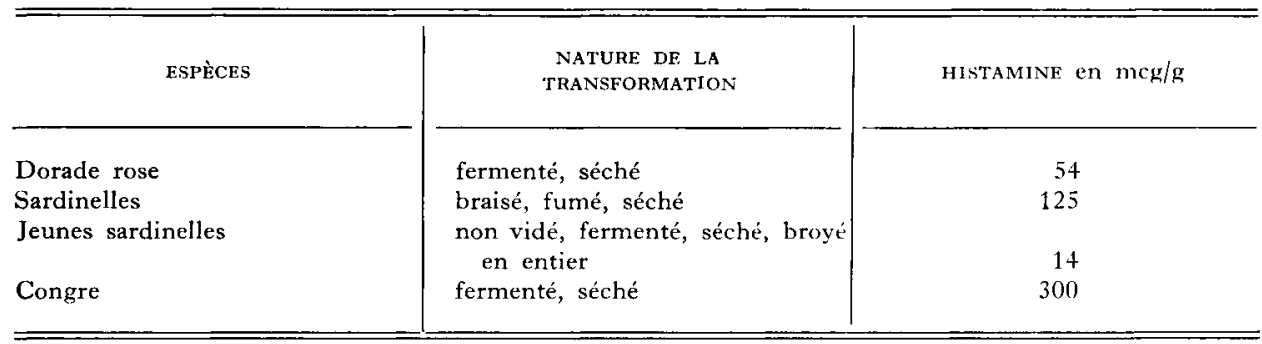

Ces échantillons proviennent de produits bien préparés (moins de $20 \%$ d'eau) et après deux mois de conservation. Signalons que selon p. Maurauges (6), les thons ayant provoqué des intoxications hitaminiques en France renfermeraient $3,5 \mathrm{mg} / \mathrm{g}$, c'est-à-dire plus de dix fois les taux que nous observons ici.

Tels sont les premiers résultats que nous pouvons donner. Ces études n'ont débuté que depuis quelques mois, elles sont encore à leur étude initiale.

Nous comptons prochainement aborder le problème de la lysine " utilisable».

Dans cette étude nous avons surtout voulu mettre l'accent sur les modifications qui peuvent se produire au cours du traitement traditionnel du poisson en vue de sa conservation. Ce traitement est encore empirique. Nous espérons ici apporter notre modeste contribution dans l'amélioration des produits transformés. 